\title{
Influencia de la Ingeniería de Software en los Procesos de Automatización Industrial
}

\author{
Ángel Mejía-Neira( ${ }^{(1)}$, Daladier Jabba ${ }^{(2)}$, Garyn Carrillo Caballero(2), y José Caicedo-Ortiz ${ }^{(3)}$ \\ (1) Universidad de Granada, Departamento de Lenguajes y Sistemas Informáticos, Granada - España \\ (e-mail: amneira@correo.ugr.es) \\ (2) Universidad del Norte, Departamento de Ingeniería de Sistemas, Barranquilla - Colombia \\ (e-mail: djabba@uninorte.edu.co; garync@uninorte.edu.co) \\ (3) Universidad de la Costa, Departamento de Ciencias de la Computación y Electrónica, Barranquilla - Colombia \\ (e-mail: jcaicedo1@cuc.edu.co)
}

Recibido Dic. 12, 2018; Aceptado Feb. 7, 2019; Versión final May. 1, 2019, Publicado Oct. 2019

\begin{abstract}
Resumen
La temática tratada en el documento abarca principalmente la influencia de la arquitectura orientada al servicio y de cómo los servicios se orquestan entre sí para el desarrollo de aplicaciones más robustas y dinámicas orientadas a la solución de problemáticas presentadas en el diseño de sistemas de automatización industrial. En la actualidad, las industrias están obligadas a utilizar nuevos sistemas que soporten la dinámica a la que se enfrentan las organizaciones. Sin embargo, los sistemas actuales de las empresas carecen de este dinamismo, lo que muchas veces imposibilita la implementación de nuevas funcionalidades a los procesos de producción autónomos, debido a la poca flexibilidad y agilidad de responder a la dinámica de los estilos de producción que se experimentan hoy día. La ingeniería de software aporta a la dinámica en el área de la automatización industrial, resaltando mejoras significativas en la configuración de sistemas implementados en el campo de la automatización industrial.
\end{abstract}

\section{The Influence of Software Engineering on Industrial Automation Processes}

\begin{abstract}
The topic dealt with in the document mainly covers the influence of service-oriented architecture and how services are orchestrated among themselves for the development of more robust and dynamic applications aimed at solving problems presented in the design of industrial automation systems. Currently, industries are compelling to use new systems that support the dynamics that organizations face. However, the current systems of companies lack this dynamism, which often makes it impossible to implement new functionalities to the processes of autonomous production, due to the lack of flexibility and agility to respond to the dynamics of production styles that are experienced today. Software engineering contributes to the dynamics in the area of industrial automation, highlighting significant improvements in the configuration of systems implemented in the field of industrial automation.
\end{abstract}




\section{INTRODUCCIÓN}

La industria moderna tiene como reto principal integrar las necesidades de los clientes con las distintas tecnologías de automatización utilizadas en los procesos industriales (Acharya et al., 2018); estas tecnologías permiten contar con sistemas efectivos, robustos, y autónomos de las tareas que se ejecutan en los procesos internos de las industrias. Los ciclos de producción actual se caracterizan principalmente por su ejecución eficiente con incrementos exponenciales de producción, y por la competencia que estos generan en las organizaciones; situación que ayuda a identificar la importancia de los procesos implementados en las industrias y cómo deben responder a las demandas del mercado y la efectividad que exige el entorno, siempre conservando como premisa principal el balance costo-beneficio de la solución en el entorno industrial de los proyectos (Fletcher et al., 2019).

En el campo de la automatización, algunos de los paradigmas utilizados para el diseño de sistemas para el control de procesos, son los sistemas de manufactura reconfigurables o RMS (Reconfigurable Manufacturing System, por sus siglas en inglés) son aquellos que para poder agregar una nueva funcionalidad, se debe configurar todo nuevamente, pero los cambios y las prestaciones que el nuevo sistema posea depende de las características de cada equipo (Bortolini et al., 2018; Maganha et al., 2018); y los sistemas de manufactura flexible o FMS (Flexible Manufacturing Systems, por sus siglas en inglés) (Lucas Silva et al., 2017), este paradigma dota un sistema de ciertos niveles de integración entre diversos dispositivos de distintas familias para que puedan configurarse de tal manera que permitan agregar funcionalidades sin tener que reconfigurar el sistema a nivel de dispositivos en su totalidad. Las nuevas tecnologías y la exigencia en los entornos organizacionales, permitieron el nacimiento de nuevas soluciones que permiten la integración de otros sistemas para la adquisición y procesamiento de la información.

La tendencia en los entornos industriales es crear inteligencia de sistemas por parte de una gran población de dispositivos pequeños, conectados en red y con un alto nivel de detalle en cuanto a niveles de inteligencia se refiere, facilitar la adaptabilidad y reconfiguración de los sistemas, por lo que permite satisfacer las necesidades empresariales no previstas durante la etapa de diseño, otorgando de este modo beneficios reales a nivel empresarial (Aguilar, 2017). La arquitectura orientada al servicio, SOA (Service-Oriented Architecture, por sus siglas en inglés) puede actuar como una tecnología unificadora que abarca varias capas, desde sensores y actuadores utilizados para monitoreo y control a nivel de planta, hasta sistemas empresariales y de ingeniería, así como sus procesos, como se muestra en la Figura 4. Esta red común significa que la comunicación de máquina a máquina no se limita a la interacción directa del dispositivo (por ejemplo, la proximidad), sino que incluye una amplia gama de interacciones en una capa cruzada con una variedad de dispositivos heterogéneos, así como sistemas y sus servicios de loT. Esto produce múltiples beneficios para todas las partes interesadas. Dichas visiones se han propuesto (Bicocchi et al., 2019) y se han realizado, demostrando los beneficios y desafíos involucrados (Leitão et al., 2016). Existen varios patrones sobre cómo se puede realizar dicha integración (Ritter et al., 2017), y las tecnologías desempeñan un papel clave en dicha orquestación de dispositivos y sistemas.

Las tecnologías basadas en protocolos web como, WS, REST, SOAP, entre otros, constituyen un enfoque prometedor para la integración (Han y Crespi, 2017), incluida el siguiente nivel en la industria, conocida como Industria 4.0 (Alcácer y Cruz-Machado, 2019). Además, los requisitos específicos de la industria para la seguridad, la capacidad de recuperación y la disponibilidad de información de eventos en tiempo casi real deben abordarse de manera efectiva. Estos últimos también son vistos como habilitadores claves para un enfoque más en tiempo real hacia la interacción con los sistemas y aplicaciones empresariales, como la monitorización de la actividad en tiempo real, la optimización de recursos y operatividad de equipos, así como la del mantenimiento.

\section{SISTEMAS DE AUTOMATIZACIÓN ACTUALES}

Las industrias hoy día, están obligadas a utilizar nuevos sistemas que soporten la dinámica a la que se enfrentan las organizaciones, sin embargo, los sistemas actuales de las empresas carecen de este dinamismo, lo que muchas veces imposibilita la implementación de nuevas funcionalidades a los procesos de producción autónomos, debido a la poca flexibilidad y agilidad de responder a la dinámica de los estilos de producción que se experimentan hoy día (Koren, et al., 2018). La evolución de las tecnologías de la información, ha incrementado la posibilidad de tener una nueva visión acerca de los procesos de manufactura en el área de la automatización industrial, cuyo reto se centra en la flexibilidad y reconfiguración de éstos. La figura 1, muestra cómo se encuentran configurados alguno de los sistemas de automatización en la actualidad (Alphonsus y Abdullah, 2016).

Actualmente, existen algunos paradigmas que ayudan a afrontar los retos que pueden llegar a presentarse en el área de la automatización industrial; uno de estos paradigmas son los sistemas holónicos de 
manufactura (Papp et al., 2018), cuya característica permite que los componentes de fábrica sean modelados como holones, los cuales son entidades de carácter autónomo y que cooperan entre sí, permitiendo desarrollar sistemas de producción más eficiente, con mayor resistencia a perturbaciones que puedan presentarse, con una facilidad de adaptación a los cambios y sobre todo más robustos.

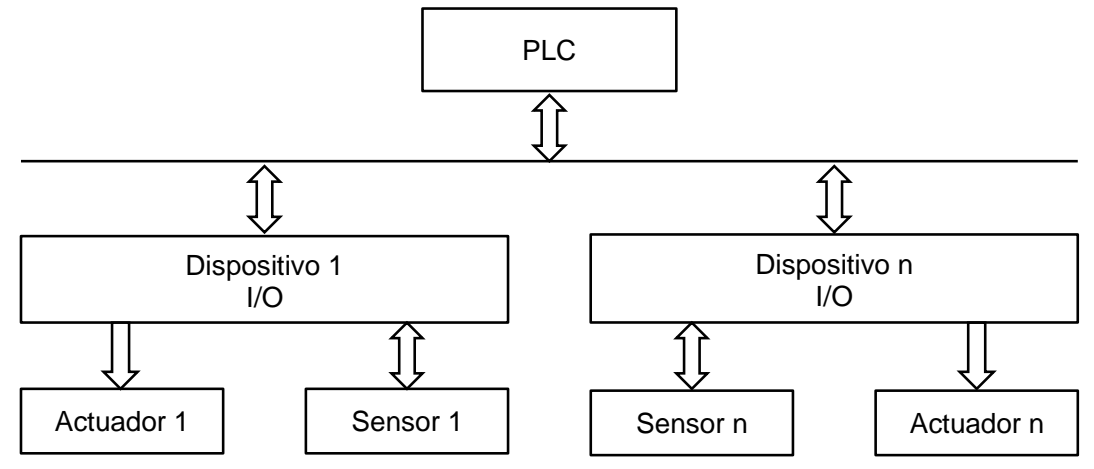

Fig. 1: Sistemas de automatización.

El paradigma descrito previamente, es establecido una vez se definen las estructuras distribuidas, las cuales se basan en entidades autónomas distribuidas y cooperantes, que por lo general son sistemas inteligentes. Contrario a lo lanzado al mercado, las cadenas de producción, tienen un denominador común: los procesos de automatización industrial encaran un reto, el cual está enfocado en la búsqueda de nuevas formas de adopción de tecnologías, encaminadas a la mejoría de los resultados en las cadenas de producción. Un ejemplo de la implementación de mejoras tecnológicas en cadenas de producción, es la implementación del estándar IEC 61499, que consiste en bloques de funciones que recortan las limitaciones de otros estándares en términos de modularidad y distribución, en (Giret et al., 2016; Jetley et al., 2013; Mendes et al., 2009 y Vyatkin, 2013).

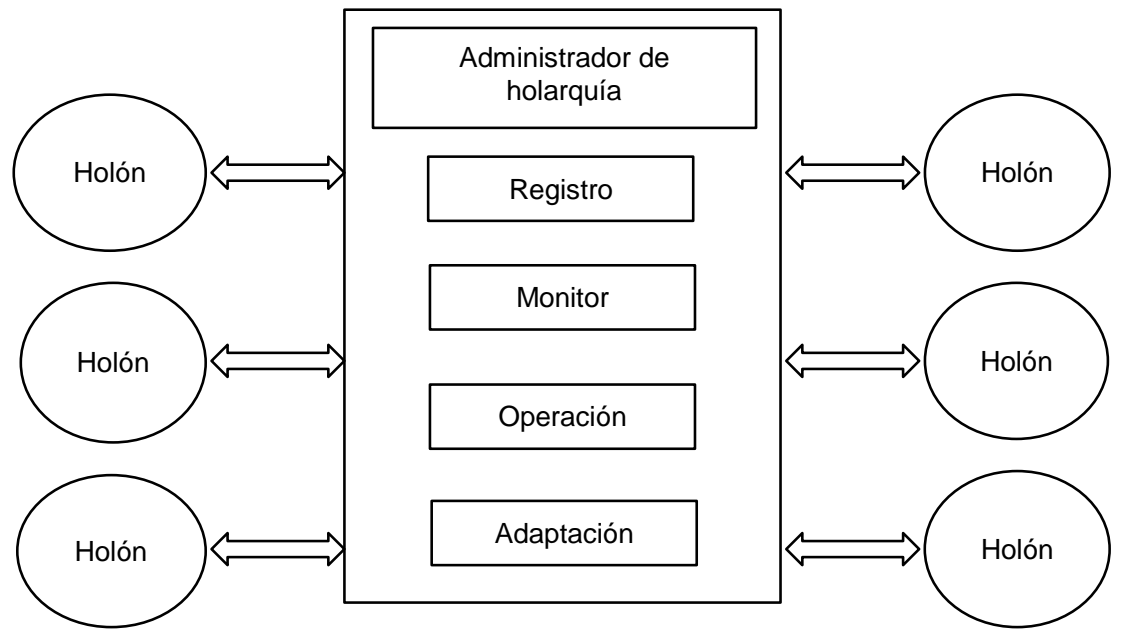

Fig.2: Arquitectura de un sistema Holónico (Adaptada de Papp et al., 2018; Essers y Vaneker, 2016)

\section{ANTECEDENTES}

Las empresas de manufactura enfrentan un entorno cambiante hoy día, sobre todo, experimentan presión en cuanto a los tiempos de salida al mercado de nuevos productos, en donde la tecnología se va incrementando y de paso la competencia. Por lo que una de las estrategias que se deben plantear al interior de las empresas debería centrarse en cómo mantener ese ímpetu competitivo, con el fin de volverse negocios altamente exitosos, ya sea a través de la fabricación de nuevos productos o innovar a partir de otros. Teniendo en cuenta lo anteriormente descrito y basado en la naturaleza del comportamiento del mercado, se describen algunos de los componentes relevantes para las organizaciones a tener en cuenta: Tener una comunicación entre los diversos niveles de la empresa; Incorporación de capital humano; Proporcionar tolerancia a fallos a aquellos subsistemas, los cuales posibiliten detección de fallos y recuperación de estos, con el fin de minimizar el impacto negativo que se puedan llegar a presentar en el ambiente de trabajo; E Incorporar a los sistemas la capacidad de ser reconfigurables, ágiles y escalables.

En el inicio de la era de la automatización industrial, el diseño de sistemas automáticos para el control de procesos se caracterizaba por el diseño de una arquitectura centralizada y jerárquica, basado en 
controladores lógicos programables o PLC (Programmable Logic Controller, por sus siglas en inglés) donde se tenía el control de todo el proceso (Fig. 1). Sin embargo, con el pasar del tiempo y la aparición de nuevas y mejoradas tecnologías y herramientas han permitido que este tipo de diseño hayan sido mejorados en favor de tener sistemas más robustos y dinámicos, como lo son los sistemas holónicos, donde cada uno de los componentes tienen funciones definidas en pro del funcionamiento del sistema, facilitando la dinámica de interacción entre ellos y el proceso (Papp et al., 2018). Actualmente, es sabido que hay ciertos requisitos que pueden satisfacerse en diversos niveles de la industrial, mediante nuevas tecnologías, una de ellas es la adopción de SOA, la cual permite tener una única comunicación basada en un solo paradigma. Aunque el reto se centra inicialmente en hacer que los ingenieros de automatización adquieran esta nueva filosofía de servicio orientada a la arquitectura (Jammes y Smit, 2005). Hoy día, el uso de los paradigmas utilizados en la ingeniería de software al campo de la automatización industrial no es muy elevado, debido al cambio de la filosofía de programación que esto implica en los ingenieros de automatización (Akdur et al., 2018).

\section{Del modelo CIM al paradigma SOA}

La necesidad de tener un mejoramiento en la calidad de la producción, así como el incremento de ésta, hizo que muchas de las empresas invirtieran en procesos que le permitieran tener un perfeccionamiento en cuanto a los procesos de manufactura, ya que pasaron de ser una simple línea de producción a una completa comunicación entre los procesos de niveles de campo y administrativos. Es ahí cuando aparece la manufactura integrada por computador o CIM (Computer Integrated Manufacturing, por sus siglas en inglés), como una de las primeras aplicaciones de las tecnologías de la información, en la que se presenta una revolución en la gestión de los procesos llevados a cabo en las industrias. Sin embargo, al comienzo de este modelo y el poco conocimiento que se tenía acerca de las tecnologías de la información, se pensaba que las empresas tendrían sus procesos automatizados, por lo que estarían desiertas y no habría necesidad del recurso humano para ejecutar los trabajos (Gopalakrishnan et al., 2014).

El modelo CIM consiste en la integración de computadoras y redes de comunicación, que constituyen un sistema de producción integrado; aunque éste prometía ser una herramienta poderosa para la implementación en la industria, debido a que esto suponía una mejora en los procesos de producción, tuvo dificultades en cuanto a la gestión de una interfaz que habilitara la comunicación de manera eficiente entre diversos puntos en todo el proceso de automatización (Delaram y Fatahi Valilai, 2018); con lo que se dio paso a la evolución de nuevos paradigmas que ayudaran a resolver este problema, como lo fueron en su momento los procesos de manufactura ágil, ingeniería concurrente, sistemas de gestión de calidad (Brandl et al, 2018). Los sistemas de automatización, se representan por la pirámide CIM, la cual permite visualizar la estructura jerárquica los sistemas y dispositivos que integran cada uno de los niveles en automatización industrial, que son: los sistemas de planificación de recursos empresariales-ERP (Enterprise Resource Planning, por sus siglas en inglés) (Pinto et al., 2017), los sistemas de ejecución de fabricación-MES (Manufacturing Execution Systems, por sus siglas en inglés) y piso de planta, donde se encuentran los dispositivos de campo y los sistemas de control (Zernadji et al., 2016).

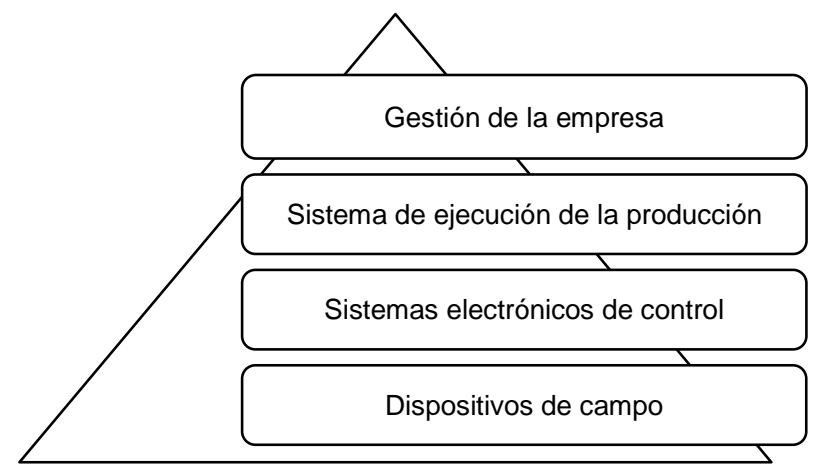

Fig. 3: Pirámide CIM (Adaptada de Carmona et al., 2016)

SOA es una colección de servicios e infraestructura para la comunicación entre ellos, que puede llegar a consistir en funciones tan sencillas como el envío de mensajes o tan complejas como la coordinación de servicios que pueden ser ejecutados en capas superiores. Un servicio es una función definida (siempre proporciona una funcionalidad independiente de otros servicios) (Kacem et al., 2012) y que es independiente de los otros servicios, éstos pueden combinarse de manera dinámica en tiempo de aplicaciones para construir aplicaciones compuestas ya sea ensamblando aplicaciones que ya estuviesen creadas o empezar con nuevas aplicaciones. A este proceso se le conoce como orquestación y coreografía. 
SOA no sólo proporciona dinamismo a procesos, sino que ayuda a solucionar problemas asociados a la escalabilidad y flexibilidad de los sistemas (Waris et al., 2013), debido a la creación de eficiencia y reusabilidad de los programas previamente desarrollados (Schmidt et al., 2018; Zeller y Weyrich, 2018). En la actualidad, existen diversos paradigmas entre los cuales se encuentra la programación orientada a objetos y la programación basada en componentes, que en conjunto con la arquitectura orientada al servicio pueden ayudar a mejorar el software que se diseña para los programas de automatización. Hoy en día, tanto el tamaño físico como las funcionalidades de las plantas están aumentando y calcular el poder computacional entregado por los autómatas no coincide con los esfuerzos que éstos realizan. A menudo, resulta poco eficiente tener un control de sistemas automáticos controlados por un solo PLC, en lugar de ellos se obtendría un mejor rendimiento si toda la lógica de se distribuyera entre varios PLC que colaboraran entre sí (El Zaatari et al., 2019; Morel et al., 2019). Sin embargo, la arquitectura de la norma IEC 61131-3 no se ajusta a la arquitectura de un sistema distribuido. Para hacer frente a las deficiencias de la norma IEC 61131-3, en el año 2005 fue publicado el estándar IEC 61499 (Jovanović et al., 2016), con el fin de ayudar al desarrollo de los sistemas de automatización distribuidas.

La norma IEC 61499 allana el camino para el uso práctico de diseño basado en componentes de software en el dominio de la automatización industrial, como se muestra en (Dai et al., 2013). Tal como se define en la norma IEC 61499, toda la lógica de control debe ser encapsulada en bloques de función. Un bloque de función es un componente de software, lo que podría ser reutilizado por el mapeo de su interfaz. El paradigma propone iniciar la programación de una librería estándar en lugar de iniciar todos los programas desde cero. Los programas de automatización pueden ser generados a partir de la configuración de los componentes de software que se requieran (Campanelli et al., 2015; Dai et al., 2015; Mazzolini et al., 2017). Sin embargo, la gestión una librería de software grande, también supone un reto. Además de esto, cabe recordar que los programas deben ser reconfigurados cuando se requiera de alguna modificación.

Con el fin de mejorar la flexibilidad y la reutilización de los programas de automatización distribuida, la arquitectura orientada a servicios es considerada como el futuro de la automatización industrial basado en la nube (Tsiatsis et al., 2019). La facilidad de acoplamiento en los componentes de software utilizados en SOA, asegura la flexibilidad e interoperabilidad entre los diversos componentes que integran la arquitectura, además, en SOA la descripción de un proceso puede ser integrado con la lógica de control, de esta manera, la descripción del proceso proporciona un mejor entendimiento del sistema y mejora la productividad del diseñador.

\section{Programación en automatización industrial}

A medida que se incrementó la complejidad de los sistemas de automatización industrial, la forma de programar software para dichos fines, presentó dos evoluciones: la lógica de control se implementaba a partir de relés, temporizadores y lazos de control cerrados, los diseños basados en esta lógica solían ser muy costosos de implementar, así como el tiempo que se necesitaba para implementarla era demasiado alto; por otra parte, se encuentran los lenguajes propios para los PLC. En 1990, nace la lógica de escalera que se asemeja a un diagrama esquemático de la lógica del relé físico, fue creada para los programas de automatización de propósito general. En 1993, la primera edición del estándar internacional para PLC IEC 61131-3 fue publicado y la tercera edición lanzada en 2013. En este estándar, se definen cuatro lenguajes de programación: lógica de escalera, estructura del texto, diagrama de instrucciones de lista y diagrama de bloques de funciones, además de una máquina de estado basada en un diagrama secuencial de estado, las unidades de organización de programación o POU (Program Organization Unit, por sus siglas en inglés) y los bloques de función también se introducen en el estándar IEC 61131-3. Como resultado, la lógica de control podría ser encapsulada en POU y ser reutilizada para el futuro. La eficiencia de diseño de software para los controladores lógicos programables, ha mejorado considerablemente mediante el uso de esos lenguajes y POU. También se introduce programación orientada a objetos para PLC en la última edición de la norma IEC 61131-3 (Jamro y Rzonca, 2018).

Los métodos para la programación en el área de la automatización industrial, han tenido una fuerte influencia en la ingeniería de software actual (Akdur et al., 2018), una de las tendencias es la programación orientada a objetos, la cual se está incluyendo en los métodos de programación actuales. Los objetos son definidos como entidades de software que encapsulan información y métodos para el procesamiento de ésta. En la automatización industrial, los objetos de software, hacen referencia a objetos físicos (dispositivos), como lo actuadores, sensores, entre otros (Gröhn et al., 2017). Estos son controlados por señales que son generadas desde otros dispositivos o a través de una cadena de invocación por parte de otros objetos.

Otro de los enfoques utilizados en la programación en automatización, es el basado en componentes, definido en el estándar IEC 61499, el cual está basado en bloques de funciones, los cuales permiten modelar los componentes. Los bloques de función están conectados a otros bloques de funciones con interfaces 
predefinidas. De igual manera, un componente puede componerse de otros componentes, con lo que se habilita el modelamiento jerárquico de estructuras que pueden ayudar a reusar el diseño de subsistemas que han sido elaborados previamente.

Con la comunicación Ethernet aplicada a nivel de planta, la arquitectura orientada a servicio se proyecta como un marco de trabajo ideal para ser aplicado en los sistemas de automatización distribuidos. Como se ha mencionado anteriormente, SOA es un conjunto de componentes, cuyas interfaces pueden ser publicadas y descubiertas. Los proveedores de servicio, así como los consumidores están acoplados con el fin de asegurar un mínimo de dependencias entre servicios. La interacción entre los servicios está definida en el contrato, el cual puede ser publicado en el repositorio. Esta interacción, también puede ser representada por medio de artefactos definidos en el estándar IEC 61499. Para asegurar el acoplamiento de los dispositivos en los programas elaborados bajo este estándar los bloques deben encapsular la mayoría de las funcionalidades, las cuales sólo requieren un mínimo de información externa. En la tabla 1, se muestra una comparación entre diversas arquitecturas $\mathrm{TI}$.

SOA tiene la mejor flexibilidad debido a que tiene las interfaces definidas por contratos de servicio entre unidades de software, adicionalmente, es el único enfoque que puede ser integrado con el bus de servicio empresarial a través de BPEL. ERP puede influenciar los servicios de manera directa a través del bus de servicio. Así, con el fin mejorar la flexibilidad e interoperabilidad para los sistemas automatizados distribuidos, la adopción de SOA aplicado en el nivel de diseño de software también puede ser un enfoque confiable. SIRENA (Bohn et al., 2006), fue uno de los primeros proyectos en el cual se hacía uso de SOA en el dominio de automatización industrial. Este proyecto introdujo un nivel de dispositivo de SOA basado en servicios web, denominado perfil de dispositivos para servicios web o DPWS (Device Profile Web Service, por sus siglas en inglés), donde cada dispositivo ofrece su funcionalidad como un servicio. Los servicios web apoyan el diseño de sistemas distribuidos, y están compuestos de proveedores y servicio, que ofrecen a los usuarios los cuales, hacen uso de esa funcionalidad.

La interfaz del WS, se describe en un formato legible por máquina como XML, el cual se denomina lenguaje de descripción de servicio web o WSDL (Web Service Description Language, por sus siglas en inglés), dicha descripción contiene información sobre los parámetros de los servicios y el tipo de información que devuelve. Además, se determina el formato en el que se intercambian los mensajes entre servicios. El protocolo más común utilizado para la comunicación de servicios web es SOAP (Simple Object Access Protocol, por sus siglas en inglés) sobre http (Dai et al., 2016). Una de las ventajas de los servicios web es que los servicios básicos se pueden combinar para generar nuevos servicios de orden superior. De esta manera, el proceso de producción de un producto puede componerse utilizando servicios de otras celdas de producción. A este proceso (de composición de servicios) se le conoce como orquestación, el cual es un motor que ofrece posibilidades de conectar servicios, controlar la programación de su ejecución y ofrecer una interface para los servicios recientemente creados en capas superiores, el lenguaje más utilizado para dicho fin es el BPEL (Puttonen et al., 2008).

El enfoque a nivel de campo utilizando SOA, ayuda a simplificar la reconfiguración de los sistemas de producción, lo único que el diseñador debería hacer es cambiar la lógica de orquestación, por lo que no es necesario ahondar en los detalles del intercambio de datos y comunicación. Para el mejoramiento de la funcionalidad PnP (Plug and Produce, por sus siglas en inglés), el sistema debe estar preparado para reaccionar a los cambios, por lo que se necesitan métodos de descripción sintácticos, como lenguaje de descripción de servicios web o WSDL (Web Services Description Language, por sus siglas en inglés) y el lenguaje de ejecución de procesos de negocio o BPEL (Business Process Execution Language, por sus siglas en inglés), éstos no describen el significado de la funcionalidad, que es necesaria para la orquestación del sistema, para resolver este inconveniente se utiliza semántica de servicios web, como anotaciones semánticas para WSDL o SAWSDL (Semantic Anotations for WSDL, por sus siglas en inglés) o la ontología de modelamiento de servicios web o WSMO (Web Service Modeling Ontology, por sus siglas en inglés), la semántica describe el significado del servicio y la relación entre los componentes de producción y su servicio. Para combinar estos servicios, en un sistema completamente funcional en un proceso de producción, el orquestador debe tener una base de conocimiento de ontología, la cual contiene información de los dispositivos que están disponibles, así como su localización y dependencia. Aunque el enfoque de SOA resulta un enfoque atractivo para el área de la automatización industrial, aún no está consolidado en la práctica, debido a algunas barreras en cuanto a las tecnologías y la apropiación de éstas por parte de los ingenieros de automatización (Colombo et al., 2010).

Desde el punto de vista tecnológico, algunos de los dispositivos utilizados en automatización industrial tienen un nivel de procesamiento insuficiente en cuanto a los protocolos de comunicación, aunque es un inconveniente que puede resolverse a medida que las tecnologías van mejorando; por la parte humana, en el 
área de automatización ya hay una filosofía establecida, por lo que se presenta una actitud conservadora por parte de los usuarios en cuanto a la implementación de nuevas tecnologías.

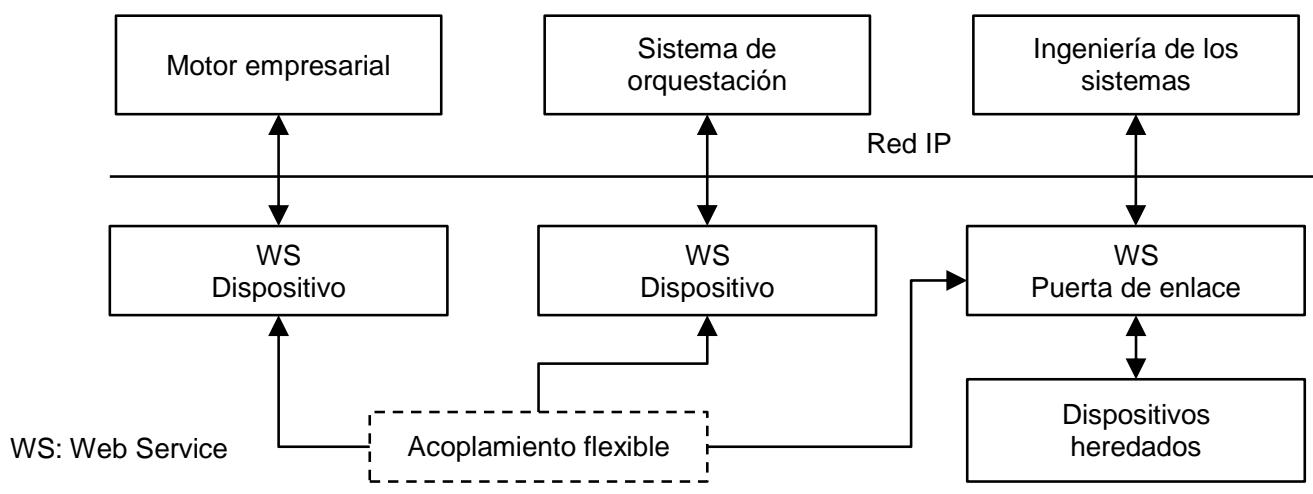

Fig. 4: Sistema de automatización basado en SOA.

\section{DISCUSIÓN FINAL}

La implementación de nuevas tecnologías en entornos industriales, trae grandes beneficios en la era industrial, sin embargo, la falta de apropiación de nuevas filosofías en los ingenieros de automatización hace que la transición a estos nuevos paradigmas sea ejecutada de manera progresiva, con el fin de asegurar que los dispositivos dentro de los diversos niveles dentro de una cadena valor no se vean afectados. Por otro lado, las soluciones flexibles y reutilizables que son implementadas a través de SOA son productos de una arquitectura de software y no sólo debe concebirse como la utilización de una herramienta, lo que conlleva a la implementación de diversos estándares, así como cambios organizacionales y culturales de la empresa. Así mismo, con la inclusión de estos paradigmas, los sistemas de producción actuales pasarán de ser centralizados, a sistemas descentralizados e inteligentes, donde cada uno de los componentes tiene capacidades de procesamiento, comunicación, control y acción sobre los eventos que se estén ejecutando, de esta manera, cada una de las máquinas se adapta a los cambios a través de la auto-reconfiguración y optimización de funciones y recursos acorde a las órdenes provenientes del entorno o servicios configurables.

Tabla 1: Programación orientada a objeto (POO), el diseño basado en componentes (DBC) y la arquitectura orientada al servicio (SOA) aplicadas en el área de la automatización industrial.

\begin{tabular}{|c|c|c|c|c|c|c|}
\hline $\begin{array}{c}\text { Tipo de } \\
\text { arquitectura TI }\end{array}$ & $\begin{array}{l}\text { Unidad de } \\
\text { software }\end{array}$ & $\begin{array}{c}\text { Característic } \\
\text { as }\end{array}$ & Interfaz & $\begin{array}{l}\text { Fuente } \\
\text { reusable }\end{array}$ & $\begin{array}{c}\text { Jerarquía del } \\
\text { sistema }\end{array}$ & Meta-modelo \\
\hline POO & Clases & $\begin{array}{c}\text { Herencia, } \\
\text { polimorfismo }\end{array}$ & $\begin{array}{l}\text { Llamado } \\
\text { de método }\end{array}$ & $\begin{array}{c}\text { Librería de } \\
\text { objetos }\end{array}$ & $\begin{array}{c}\text { Clases } \\
\text { anidadas }\end{array}$ & $\begin{array}{l}\text { Diagramas de } \\
\text { clases (UML) }\end{array}$ \\
\hline DCB & $\begin{array}{l}\text { Componente } \\
\text { de software }\end{array}$ & $\begin{array}{c}\text { Encapsulaci } \\
\text { ón }\end{array}$ & $\begin{array}{c}\text { Interfaz } \\
\text { predefinida }\end{array}$ & $\begin{array}{c}\text { Librería de } \\
\text { componentes }\end{array}$ & $\begin{array}{c}\text { Componentes } \\
\text { anidados }\end{array}$ & $\begin{array}{c}\text { Redes de } \\
\text { componentes }\end{array}$ \\
\hline SOA & $\begin{array}{l}\text { Servicio de } \\
\text { software }\end{array}$ & $\begin{array}{c}\text { Acoplamient } \\
\text { o, } \\
\text { descubrimie } \\
\text { nto }\end{array}$ & $\begin{array}{l}\text { Contrato } \\
\text { de servicio }\end{array}$ & $\begin{array}{l}\text { Repositorio de } \\
\text { servicio }\end{array}$ & $\begin{array}{l}\text { Servicio de } \\
\text { orquestación, } \\
\text { composición }\end{array}$ & $\begin{array}{l}\text { Lenguaje de } \\
\text { ejecución del } \\
\text { proceso de } \\
\text { negocio } \\
\text { (BPEL), } \\
\text { diagrama de } \\
\text { servicio de } \\
\text { secuencia }\end{array}$ \\
\hline
\end{tabular}

\section{CONCLUSIONES}

De acuerdo al trabajo presentado y a los resultados obtenidos, se pueden plantear las siguientes conclusiones principales:

La prevalencia de dispositivos integrados avanzados, junto con mayores capacidades de procesamiento y comunicación, está transformando la automatización industrial; en donde los enfoques de arquitectura orientada a servicios ahora son posibles a nivel de dispositivo y traen consigo beneficios que anteriormente solo estaban disponibles para los diseñadores y gerentes de sistemas empresariales.

Existe una tendencia hacia la integración basada en la información que potencia las interacciones, que dan paso a la implementación de nuevas tecnologías en la industria, así como la integración con los sistemas empresariales y los respectivos procesos de negocios. 
Las tecnologías basadas en protocolos web, constituyen un enfoque prometedor para la integración. Estas tecnologías aplicadas en el campo de la automatización industrial actúan como un facilitador hacia el objetivo fundamental de permitir la fácil integración de los servicios a nivel de dispositivo con los sistemas empresariales, superando la heterogeneidad y la implementación específica de hardware y software del dispositivo.

\section{REFERENCIAS}

Acharya, V., S.K. Sharma y S. Kumar Gupta, Analyzing the factors in industrial automation using analytic hierarchy process, doi: 10.1016/J.COMPELECENG.2017.08.015, Computers y Electrical Engineering, 71, 877-886 (2018)

Akdur, D., V. Garousi y O. Demirörs, A survey on modeling and model-driven engineering practices in the embedded software industry, doi: 10.1016/J.SYSARC.2018.09.007, Journal of Systems Architecture, 91, 62-82 (2018)

Alcácer, V. y V. Cruz-Machado, Scanning the Industry 4.0: A Literature Review on Technologies for Manufacturing Systems, doi:10.1016/J.JESTCH.2019.01.006, Engineering Science and Technology, an International Journal, 2 -21 (2019)

Alphonsus, E.R. y M.O. Abdullah, A review on the applications of programmable logic controllers (PLCs), doi:/10.1016/j.rser.2016.01.025, Renewable and Sustainable Energy Reviews, 60, 1185-1205 (2016)

Bicocchi, N., G. Cabri, F. Mandreoli y M. Mecella, Dynamic digital factories for agile supply chains: An architectural approach, doi: 10.1016/J.JII.2019.02.001, Journal of Industrial Information Integration (2019)

Bohn, H., A. Bobek y F. Golatowski, SIRENA - Service Infrastructure for Real-time Embedded Networked Devices: A service-oriented framework for different domains, doi:10.1109/ICNICONSMCL.2006.196, International Conference on Networking, International Conference on Systems and International Conference on Mobile Communications and Learning Technologies, ICN/ICONS/MCL'06 (2006)

Bortolini, M., F. G. Galizia y C. Mora, Reconfigurable manufacturing systems: Literature review and research trend, doi: 10.1016/j.jmsy.2018.09.005, Journal of Manufacturing Systems (2018)

Brandl, F.J., M. Kagerer y G. Reinhart, A Hybrid Innovation Management Framework for Manufacturing - Enablers for more Agility in Plants, doi: 10.1016/J.PROCIR.2018.04.022, Procedia CIRP, 72, 1154-1159 (2018)

Campanelli, S., P. Foglia y C.A. Prete, An architecture to integrate IEC 61131-3 systems in an IEC 61499 distributed solution, doi: 10.1016/J.COMPIND.2015.04.002, Computers in Industry, 72, 47-67 (2015)

Carmona, J.A.R., J.C.M. Benitez y J.L. Garcia-Gervacio, SCADA system design: A proposal for optimizing a production line, doi:10.1109/CONIELECOMP.2016.7438574, International Conference on Electronics, Communications and Computers (CONIELECOMP), 192-197 (2016)

Colombo, A.W., S. Karnouskos y J.M. Mendes, Factory of the Future: A Service-oriented System of Modular, Dynamic Reconfigurable and Collaborative Systems, doi: 10.1007/978-1-84996-119-6_15, Artificial Intelligence Techniques for Networked Manufacturing Enterprises Management, 459-481 (2010)

Dai, W., V. Vyatkin, J.H. Christensen y V.N. Dubinin, Bridging Service-Oriented Architecture and IEC 61499 for Flexibility and Interoperability, doi: 10.1109/TII.2015.2423495, IEEE Transactions on Industrial Informatics, 11(3), 771-781 (2015)

Dai, W., Wanqi Huang y V. Vyatkin, Enabling plug-and-play software components in industrial cyber-physical systems by adopting service-oriented architecture paradigm, doi:10.1109/IECON.2016.7793834, In IECON 2016 - 42nd Annual Conference of the IEEE Industrial Electronics Society, 5253-5258 (2016)

Delaram, J. y O. Fatahi Valilai, An architectural view to computer integrated manufacturing systems based on Axiomatic Design Theory, doi: 10.1016/J.COMPIND.2018.04.009, Computers in Industry, 100, 96-114 (2018)

El Zaatari, S., M. Marei, W. Li y Z. Usman, Cobot programming for collaborative industrial tasks: An overview, doi: 10.1016/j.robot.2019.03.003, Robotics and Autonomous Systems (2019)

Essers, M.S. y T.H.J. Vaneker, Design of a decentralized modular architecture for flexible and extensible production systems, doi: 10.1016/J.MECHATRONICS.2015.08.009, Mechatronics, 34, 160-169 (2016)

Fletcher, S., T. Johnson y otros cinco autores, Adaptive Automation Assembly: Identifying System Requirements for Technical Efficiency and Worker Satisfaction, doi: 10.1016/J.CIE.2019.03.036, Computers y Industrial Engineering (2019)

Giret, A., E. Garcia y V. Botti, An engineering framework for Service-Oriented Intelligent Manufacturing Systems, doi: 10.1016/j.compind.2016.02.002, Computers in Industry, 81, 116-127 (2016)

Gopalakrishnan, A., K.P. Jithin y A.C. Biswal, Industrial Automation System on Device (IndASoD) -A device modeling industrial automation scenarios, doi:10.1109/INDICON.2014.7030500, Annual IEEE India Conference (INDICON), 1-6 (2014)

Gröhn, L., S. Metsälä y otros cinco autores, Manufacturing System Upgrade with Wireless and Distributed Automation, doi: 10.1016/j.promfg.2017.07.207, Procedia Manufacturing, 11, 1012-1018 (2017)

Han, S.N. y N. Crespi, Semantic service provisioning for smart objects: Integrating loT applications into the web, doi: 10.1016/J.FUTURE.2016.12.037, Future Generation Computer Systems, 76, 180-197 (2017)

Jammes, F., y H. Smit, Service-oriented paradigms in industrial automation, doi: 10.1109/TII.2005.844419, IEEE Transactions on Industrial Informatics, 1(1), 62-70 (2005) 
Jamro, M. y D. Rzonca, Agile and hierarchical round-trip engineering of IEC 61131-3 control software, doi: 10.1016/j.compind.2018.01.004, Computers in Industry, 96, 1-9 (2018)

Jetley, R., A. Nair, P. Chandrasekaran y A. Dubey, Applying software engineering practices for development of industrial automation applications, doi:10.1109/INDIN.2013.6622945, IEEE International Conference on Industrial Informatics (INDIN), 558-563 (2013)

Jovanović, M., S. Zupan e I. Prebil, Holonic control approach for the "green"-tyre manufacturing system using IEC 61499 standard, doi:10.1016/J.JMSY.2016.06.008, Journal of Manufacturing Systems, 40, 119-136 (2016)

Joyanes Aguilar, L., La transformación digital en las organizaciones y empresas, Industria 4.0 la cuarta revolución industrial, Alfaomega, 73-95, Bogotá, Colombia (2017)

Kacem, H.H., W. Sellami y A.H. Kacem, A formal approach for the validation of web service orchestrations, doi: 10.1109/WETICE.2012.53, Proceedings of the Workshop on Enabling Technologies: Infrastructure for Collaborative Enterprises, WETICE, 42-47 (2012)

Koren, Y., X. Gu y W. Guo, Reconfigurable manufacturing systems: Principles, design, and future trends, doi: 10.1007/s11465-018-0483-0, Frontiers of Mechanical Engineering, 13(2), 121-136 (2018)

Leitão, P., A.W. Colombo y S. Karnouskos, Industrial automation based on cyber-physical systems technologies: Prototype implementations and challenges, doi: 10.1016/j.compind.2015.08.004, Computers in Industry, 81, 11-25 (2016)

Lucas Silva, A., R. Ribeiro y M. Teixeira, Modeling and control of flexible context-dependent manufacturing systems, doi: 10.1016/j.ins.2017.08.084, Information Sciences, 421, 1-14 (2017)

Maganha, I., C. Silva y L.M.D.F Ferreira, Understanding reconfigurability of manufacturing systems: An empirical analysis, doi: 10.1016/j.jmsy.2018.07.004, Journal of Manufacturing Systems, 48 (Part A), 120-130 (2018)

Mazzolini, M., F.A. Cavadini, G. Montalbano y A. Forni, Structured Approach to the Design of Automation Systems through IEC 61499 Standard, doi:10.1016/J.PROMFG.2017.07.194, Procedia Manufacturing, 11, 905-913 (2017)

Mendes, J.M., A. Bepperling y otros cuatro autores, Software methodologies for the engineering of service-oriented industrial automation: The continuum project, doi: 10.1109/COMPSAC.2009.66, Proceedings - International Computer Software and Applications Conference, 1, $452-459$ (2009)

Morel, G., C.E. Pereira y S.Y. Nof, Historical survey and emerging challenges of manufacturing automation modeling and control: A systems architecting perspective, doi: 10.1016/j.arcontrol.2019.01.002, Annual Reviews in Control (2019)

Papp, J., D. Tokody y F. Flammini, From traditional manufacturing and automation systems to holonic intelligent systems, doi: 10.1016/j.promfg.2018.03.132, Procedia Manufacturing, 22, 931-935 (2018)

Pinto, S.M., P.E. Ramírez y E.E. Grandón, Antecedentes del Éxito de los Sistemas de Planificación de Recursos Empresariales en las Grandes Empresas Chilenas: un Modelo Factorial Exploratorio, doi:10.4067/S071807642017000300015, Información Tecnológica, 28(3), 139-146 (2017)

Puttonen, J., A. Lobov y J.L.M. Lastra, An application of BPEL for service orchestration in an industrial environment, doi: 10.1109/ETFA.2008.4638450, IEEE International Conference on Emerging Technologies and Factory Automation, 530$537(2008)$

Ritter, D., N. May y S. Rinderle-Ma, Patterns for emerging application integration scenarios: A survey, doi: 10.1016/J.IS.2017.03.003, Information Systems, 67, 36-57 (2017)

Schmidt, J. P., T. Müller y M. Weyrich, Methodology for the model driven development of service oriented plant controls, doi: 10.1016/j.procir.2017.12.195, Procedia CIRP, 67, 173-178 (2018)

Tsiatsis, V., S. Karnouskos y otros tres autores, Industrial Automation, doi: 10.1016/B978-0-12-814435-0.00024-9, Internet of Things, 249-256 (2019)

Vyatkin, V., Software Engineering in Industrial Automation: State-of-the-Art Review, doi: 10.1109/TII.2013.2258165, IEEE Transactions on Industrial Informatics, 9(3), 1234-1249 (2013)

Waris, M., S.A. Khan y M.Z. Fakhar, Factors effecting service-oriented architecture implementation, Proceedings of 2013 Science and Information Conference, ISBN: 978-0-9893193-0-0, 1-8 (2013)

Zeller, A. y M. Weyrich, Component based Verification of Distributed Automation Systems based on Model Composition, doi: 10.1016/j.procir.2018.03.183, Procedia CIRP, 72, 352-358 (2018)

Zernadji, T., C. Tibermacine, F. Cherif y A. Zouioueche, Integrating quality requirements in engineering web service orchestrations, doi: 10.1016/J.JSS.2015.11.009, Journal of Systems and Software, 122, 463-483 (2016) 
